\title{
Characterization study of GaN-based epitaxial layer and light-emitting diode on nature-patterned sapphire substrate
}

\author{
H.Y. Lin, Y.J. Chen, C.L. Chang, and X.F. Li \\ Department of Chemical and Materials Engineering, National Central University, Jhongli City, Taiwan 32001, \\ Republic of China \\ C.H. Kuo \\ Institute of Lighting and Energy Photonics, National Chiao Tung University, Tainan City, Taiwan 30010, \\ Republic of China \\ S.C. Hsu \\ Department of Chemical and Materials Engineering, Tamkang University, New Taipei City, Taiwan 25137, \\ Republic of China \\ C.Y. Liu ${ }^{\text {a) }}$ \\ Department of Chemical and Materials Engineering, National Central University, Jhongli City, Taiwan 32001, \\ Republic of China
}

(Received 14 October 2011; accepted 28 December 2011)

\begin{abstract}
Chemical wet etching on c-plane sapphire wafers by three etching solutions $\left(\mathrm{H}_{3} \mathrm{PO}_{4}, \mathrm{H}_{2} \mathrm{SO}_{4}\right.$, and $\mathrm{H}_{3} \mathrm{PO}_{4} / \mathrm{H}_{2} \mathrm{SO}_{4}$ mixing solution) was studied. Among these etching agents, the mixing $\mathrm{H}_{3} \mathrm{PO}_{4} / \mathrm{H}_{2} \mathrm{SO}_{4}$ solution has the fastest etching rate $(1.5 \mu \mathrm{m} / \mathrm{min})$. Interestingly, we found that $\mathrm{H}_{2} \mathrm{SO}_{4}$ does not etch the c-plane sapphire wafer in thickness; instead, a facet pyramidal pattern is formed on the c-plane sapphire wafer. GaN light-emitting diode (LED) epitaxial structure was grown on the sapphire wafer with the pyramidal pattern and the standard flat sapphire wafer. X-ray diffraction and photoluminescence measurement show that the pyramidal pattern on the sapphire wafer improved crystalline quality but augmented the compressive stress level in the GaN LED epilayer. The horizontal LED chips fabricated on the pyramidal-patterned sapphire wafer have a larger light output than the horizontal LED chips fabricated on the standard flat sapphire wafer by $20 \%$.
\end{abstract}

\section{INTRODUCTION}

Nowadays, the patterned-sapphire substrate techniques have been widely used in high-power GaN-based lightemitting diodes (LEDs), which are the most promising alternative light source for general lighting. ${ }^{1,2}$ With the breakthrough of the patterned-sapphire substrate technique, the efficacy of high-brightness GaN-based LEDs has been driven to a record high of $150 \mathrm{~lm} / \mathrm{W} .^{3,4}$ The efficacy enhancement of GaN-based LEDs with the patterned-sapphire substrate technique generally attributes to the improvement in both light extraction efficiency and internal quantum efficiency. ${ }^{5-9}$ The regular patterns created on the sapphire substrate counteracts the effect of the total internal reflection at the GaN/sapphire interface. ${ }^{5}$ And, the enhancement in the internal quantum efficiency benefits from the reduction of threading dislocations by possible lateral growth of $\mathrm{GaN}$ epilayer on the patternedsapphire substrate. ${ }^{5,10-13}$

\footnotetext{
a)Address all correspondence to this author.

e-mail: chengyi@cc.ncu.edu.tw

DOI: $10.1557 / j m r .2012 .24$
}

Numerous patterning features produced on the patternedsapphire substrate by either dry etching or wet etching processes, which includes circle cavities, square cavities, hemispheric bumps, and trenched stripes, have been studied. ${ }^{5,14-16}$ Yet, no matter what etching process is used to create the patterns, a hard mask lithographic process is required on the flat c-plane sapphire wafer. In this study, a mask-free wet etching process was used to produce a naturepatterned sapphire substrate (n-pss). Then, a metalorganic chemical vapor deposition (MOCVD) GaN LED epilayer was grown on the n-pss wafer. The MOCVD GaN epilayers were characterized by photoluminescence (PL) and x-ray rocking curve analysis. The above analysis can spell out the crystalline quality and the in-plane stress level of the GaN epilayer grown on the n-pss wafer. Previous researches have shown that the residue compressive stress in the GaN epilayer would result in a piezoelectric field across the multiquantum wells (MQWs). The piezoelectric field distorts the energy levels in the energy band diagram of the MQWs of the GaN LED epilayer, which degrades the internal quantum efficiency. ${ }^{15,16}$ So, the in-plane stress level of the GaN epilayer grown on the patterned-sapphire substrate is another important factor affecting the efficacy of the GaN-based 
LED. So far, there is no systematical study on the in-plane stress of the GaN epilayer grown on the patterned sapphire substrate. How do the patterns on the patterned sapphire substrate affect the in-plane stress level of the GaN epilayer is still unknown. In this work, a comparison study would be carried on the in-plane stress level in the $\mathrm{GaN}$ epilayers grown on the n-pss wafer and on the standard sapphire wafer.

\section{EXPERIMENTAL}

The sapphire wafers studied in this work are the common 2-inch c-plane sapphire wafers. A cohesive contact between the backside of the sapphire wafers and the bottom of the growth pots in MOCVD growth chamber is very important for growing a good crystal quality of GaN epilayers. It is because that any poor contact interface between the backside of the sapphire wafers and the bottom of the growth pots caused by the scratched or rough backside of the sapphire wafers will result in temperature fluctuation on the growth surface of the sapphire wafer during MOCVD growth process. Therefore, before the etching process, a plasma-enhanced chemical vapor deposition $\mathrm{SiO}_{2}$ layer would be deposited on the backside of the sapphire wafers to protect the backside of the sapphire wafers from being etched.

The etching on sapphire wafers was done by immersing sapphire wafers into three etching solutions: pure $\mathrm{H}_{3} \mathrm{PO}_{4}$, pure $\mathrm{H}_{2} \mathrm{SO}_{4}$, and a mixing solution $\left(\mathrm{H}_{3} \mathrm{PO}_{4}\right.$ : $\left.\mathrm{H}_{2} \mathrm{SO}_{4}=3: 1\right)$. Four etching temperatures are studied, which are $200,260,280$, and $320^{\circ} \mathrm{C}$. After certain etching times, 15, 30, and $60 \mathrm{~min}$, the sapphires wafers were removed from the etching solutions to measure the etched sapphire thickness. The etched thickness is measured by following method. $\mathrm{A} \mathrm{SiO}_{2}$ layer was deposited on the sapphire substrate. Then, by using lithography, the $\mathrm{SiO}_{2}$ layer was defined as $1 \times 1 \mathrm{~cm}^{2}$ patterns. The patterned $\mathrm{SiO}_{2}$ layer serves as a hard mask to prevent sapphire from etching. So, during the etching process, the acid only etched the "exposed" sapphire area, i.e., without $\mathrm{SiO}_{2}$ hard mask on the top. Then, we used Alpha step to measure the height difference between the area with $\mathrm{SiO}_{2}$ hard mask and without $\mathrm{SiO}_{2}$ hard mask. Then, the etched thickness of the sapphire substrate can be determined. The backside $\mathrm{SiO}_{2}$ layer can be removed by hydrogen fluoride solution. After stripping the backside $\mathrm{SiO}_{2}$ layer, the etched sapphire wafers, i.e., n-pss wafers, are ready for the growth process of MOCVD GaN LED epilayer.

\section{RESULTS AND DISCUSSIONS}

\section{A. Etching rate and etching surface morphology on sapphire wafer}

Figure 1(a) shows the etched thickness of sapphire wafers with three etching solutions $\left[\mathrm{H}_{3} \mathrm{PO}_{4}, \mathrm{H}_{2} \mathrm{SO}_{4}\right.$, and
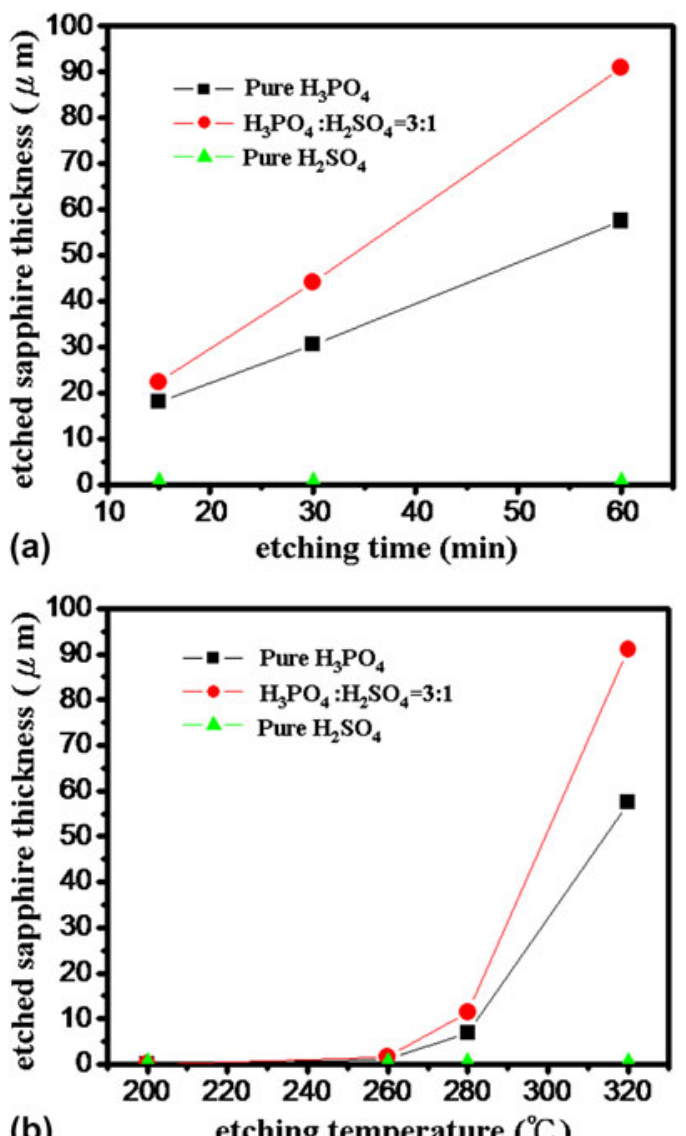

(b)

etching temperature $\left({ }^{\circ} \mathrm{C}\right)$

FIG. 1. (a) Etching thickness versus etching time at $320{ }^{\circ} \mathrm{C}$ with different solutions. (b) Etching thickness versus etching temperature for 60 min with different solutions.

the mixing $\mathrm{H}_{3} \mathrm{PO}_{4} / \mathrm{H}_{2} \mathrm{SO}_{4}$ (3:1) solution] versus the etching time at a constant temperature of $320{ }^{\circ} \mathrm{C}$. For $\mathrm{H}_{3} \mathrm{PO}_{4}$ and the mixing $\mathrm{H}_{3} \mathrm{PO}_{4} / \mathrm{H}_{2} \mathrm{SO}_{4}$ etching solutions, the etched sapphire thickness shows a very linear relationship with the etching time. The mixing $\mathrm{H}_{3} \mathrm{PO}_{4} / \mathrm{H}_{2} \mathrm{SO}_{4}$ solution has the fastest etching rate (about $1.5 \mu \mathrm{m} / \mathrm{min}$ ). Interestingly, we found that pure $\mathrm{H}_{2} \mathrm{SO}_{4}$ did not etch the sapphire wafer in thickness. Even after 60-min etching, the thickness of the sapphire wafer almost remains the same. Figure 1(b) plots the etched sapphire thickness against the etching temperatures with 60-min etching time. An onset temperature for the sapphire wafer starting to being etching is found to be about $260^{\circ} \mathrm{C}$. As the etching temperature was over $260^{\circ} \mathrm{C}$, the etching rates of both $\mathrm{H}_{3} \mathrm{PO}_{4}$ and the mixing $\mathrm{H}_{3} \mathrm{PO}_{4} / \mathrm{H}_{2} \mathrm{SO}_{4}$ etching solutions on the sapphire wafer greatly increase.

The surface morphology on the etched sapphire wafers by $\mathrm{H}_{3} \mathrm{PO}_{4}$ and the mixing $\mathrm{H}_{3} \mathrm{PO}_{4} / \mathrm{H}_{2} \mathrm{SO}_{4}$ solution is quite similar. As shown in Figs. 2(a) and 2(b), the etched sapphire surfaces by $\mathrm{H}_{3} \mathrm{PO}_{4}$ and the mixing $\mathrm{H}_{3} \mathrm{PO}_{4} / \mathrm{H}_{2} \mathrm{SO}_{4}$ solution are relatively flat and only few etching pits can be seen on the etched sapphire surface. Previously, we have shown that 
(a)

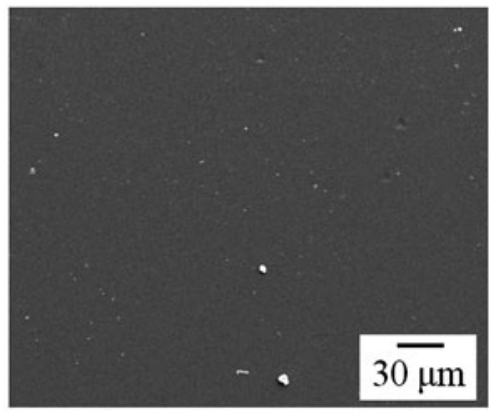

(b)

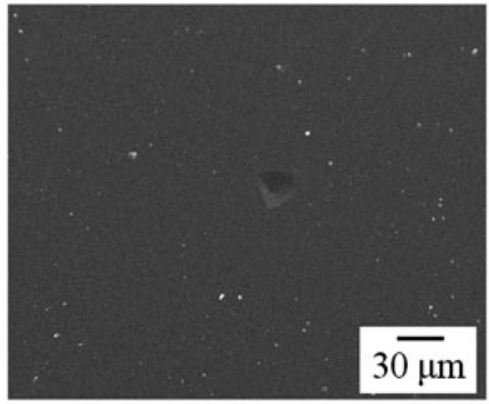

(c)
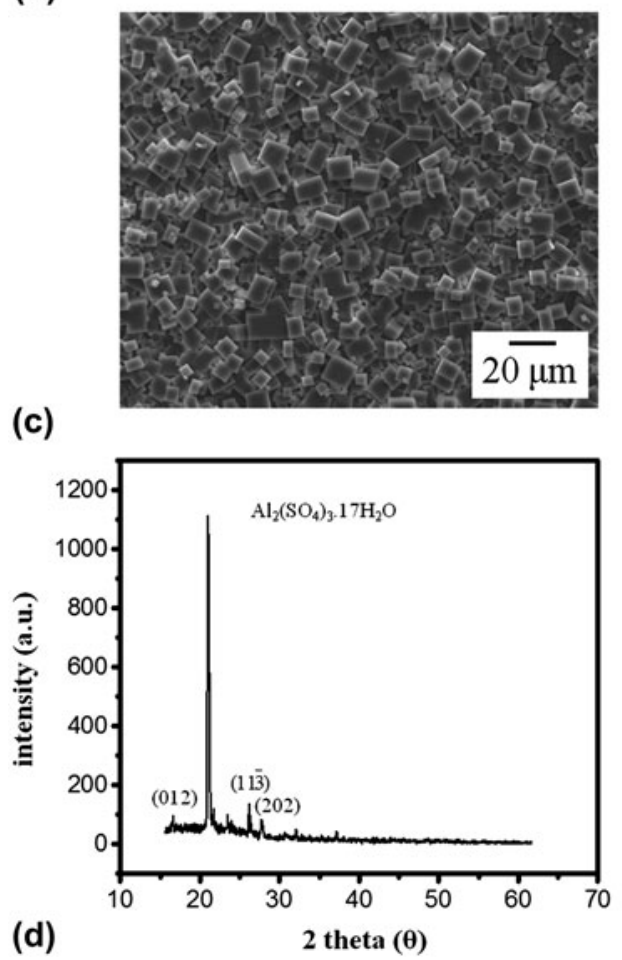

FIG. 2. (a) Morphology of etched sapphire wafer by $\mathrm{H}_{3} \mathrm{PO}_{4}$-contained solution. (b) Morphology of etched sapphire wafer by $\mathrm{H}_{3} \mathrm{PO}_{4} / \mathrm{H}_{2} \mathrm{SO}_{4}$ mixing solution. (c) Morphology of the etching reaction product by $\mathrm{H}_{2} \mathrm{SO}_{4}$. (d) X-ray diffraction (XRD) result of etching reaction product on the etched sapphire surface with $\mathrm{H}_{2} \mathrm{SO}_{4}$.

$\mathrm{H}_{2} \mathrm{SO}_{4}$ does not etch the sapphire wafer in thickness. Instead, a very dense etching reaction product phase was found and completely covered on the surface of the sapphire wafer, as seen in Fig. 2(c). The etching reaction product phase has a cubic shape. X-ray diffraction (XRD) was used to identify the exact compound phase and the structure of the etching reaction product phase. As shown in Fig. 2(d), only one strong peak appears in the XRD pattern of the etching product, which matches with the (104) plane of the standard XRD pattern of $\mathrm{Al}_{2}\left(\mathrm{SO}_{4}\right)_{3} \cdot 17 \mathrm{H}_{2} \mathrm{O}$ phase. Dwikusuma reported a similar finding on the $\mathrm{Al}_{2}$ $\left(\mathrm{SO}_{4}\right)_{3} \cdot 17 \mathrm{H}_{2} \mathrm{O}$ phase. ${ }^{14}$ However, Dwikusuma's XRD result showed a polycrystalline structure on the $\mathrm{Al}_{2}$ $\left(\mathrm{SO}_{4}\right)_{3} \cdot 17 \mathrm{H}_{2} \mathrm{O}$ etching product. In the present work, one strong (104) peak shown in XRD pattern implies that the etching product grown on the sapphire wafer should be a highly preferred orientation polycrystalline structure. The etching reaction product grew (or precipitated) with a particular $<104>$ growth direction on the c-plane of the sapphire wafer. We believe that it is the etching reaction product layer $\left(\mathrm{Al}_{2}\left(\mathrm{SO}_{4}\right)_{3} \cdot 17 \mathrm{H}_{2} \mathrm{O}\right)$ preventing the sapphire surface from being etched by $\mathrm{H}_{2} \mathrm{SO}_{4}{ }^{14,17} \mathrm{On}$ the contrary, no etching reaction product would form on the sapphire wafer as the sapphire wafer was etched by the acid solutions containing $\mathrm{H}_{3} \mathrm{PO}_{4}$, such as the $\mathrm{H}_{3} \mathrm{PO}_{4}$ or the $\mathrm{H}_{3} \mathrm{PO}_{4} / \mathrm{H}_{2} \mathrm{SO}_{4}$ mixing solution. It implies that $\mathrm{H}_{3} \mathrm{PO}_{4}$ can etch the etching reaction product phase. Thus, without forming the etching reaction product on the sapphire wafer, the sapphire wafers can be etched by $\mathrm{H}_{3} \mathrm{PO}_{4}$-contained acid solutions. ${ }^{14}$

$\mathrm{HCl}$ solution can remove the etching reaction product layer on the sapphire surface, which was resulted from the $\mathrm{H}_{2} \mathrm{SO}_{4}$ etching. After removing the etching reaction product by dilute $\mathrm{HCl}$, facet pyramids were observed on the etched sapphire surface. Figure 3(a) shows the scanning electron microscopy (SEM) image of the facet pyramids on the etched sapphire surface. The height and the width of the pyramid are about $0.2 \mu \mathrm{m}$ and 1-2 $\mu \mathrm{m}$, respectively. Energy-dispersive $\mathrm{x}$-ray diffraction spectroscopy (EDX) analysis on the pyramids on the sapphire wafer shows that no $\mathrm{S}$ atom can be found and $\mathrm{Al}$ and $\mathrm{O}$ are the only two elements that can be detected (the atomic ratio is 2:3). The EDX result is shown in Fig. 3(b). Therefore, we can confirm that the pyramids on the sapphire wafer belong to sapphire phase.

Figure 4 shows the titled SEM image on a particular pyramid on the etched sapphire surface. We can clearly see that the side-planes of the pyramids are not flat; instead, they are curved surfaces. It means that the dihedral angle of the side-planes in respect to the sapphire surface (c-plane) varies with the height of the pyramid. In the bottom part of the pyramids, the dihedral angle is relatively large and it becomes smaller toward the apex of the pyramids. So, we believe that the side-planes of the pyramids do not belong to any known common planes of the sapphire crystalline structure, such as r, m, and a planes. Those side-planes could be composed of many high-index planes of the sapphire crystalline structure. 


\section{B. Epitaxial GaN LED epilayers grown on n-pss and standard flat sapphire wafers}

Previously, it has been shown that facet pyramids can be created on the sapphire wafer by etching with $\mathrm{H}_{2} \mathrm{SO}_{4}$. The unique pyramidal morphological pattern on the sap-

(a)
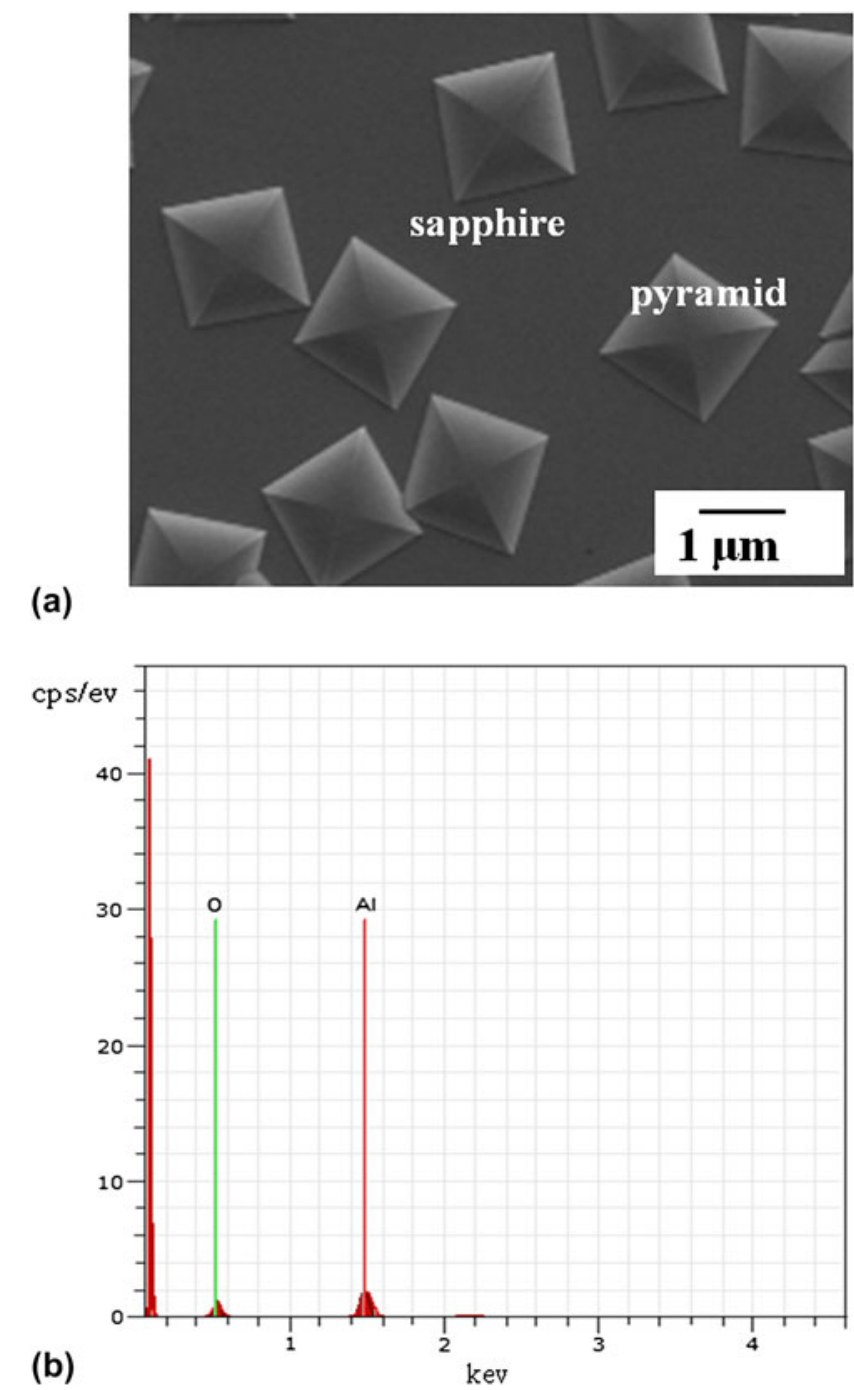

FIG. 3. (a) Scanning electron microscopy (SEM) image of the pyramids on the etched sapphire wafer. (b) Energy-dispersive $\mathrm{x}$-ray spectroscopy analysis on the pyramids on the sapphire wafer.

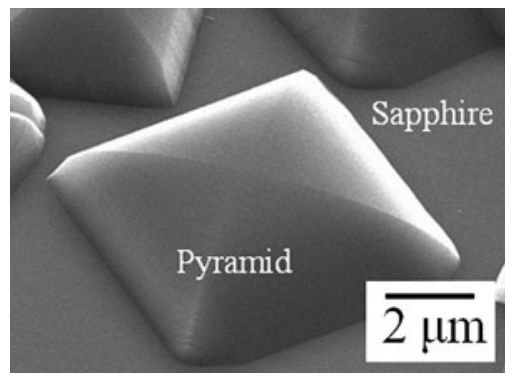

FIG. 4. Titled SEM image on a pyramid. phire surface might have similar functions with current patterned sapphire substrates, i.e., excellent improvements in the $\mathrm{GaN}$ epitaxial quality and the light-extraction efficiency for LED devices. ${ }^{5-9}$ So, it is of interesting to grow epitaxial GaN/InGaN LED structure on the n-pss wafer with the pyramidal pattern by MOCVD process. The LED epilayer structure includes a $1.8-\mu \mathrm{m}$-thick undoped $\mathrm{GaN}$ layer and a $2.5-\mu \mathrm{m}$-thick Si-doped n-type GaN cladding layer, an active region of 450-nm emitting wavelength with six periods of InGaN/GaN MQWs, and a $0.3-\mu \mathrm{m}$-thick Mg-doped p-type GaN cladding layer.

$\mathrm{X}$-ray rocking curve and PL measurement were performed on the GaN LED epilayers on the n-pss wafer and the standard flat sapphire wafer. From XRD rocking curve result [Fig. 5(a)], we can observe: (i) the (0002) diffraction peak intensity of the $\mathrm{GaN}$ epilayer on the n-pss wafer is larger than that on the standard flat sapphire wafer and (ii) also, full width at half maximum (FWHM) of the $\mathrm{GaN}$ epilayer on the n-pss wafer is narrower than that on the standard flat sapphire wafer. The above observations indicate that the crystalline quality of the GaN LED epilayer on the n-pss wafer is better than that on the standard flat sapphire wafer. In addition, the smaller FWHM of the GaN epilayer on the n-pss wafer further implies that the $\mathrm{GaN}$
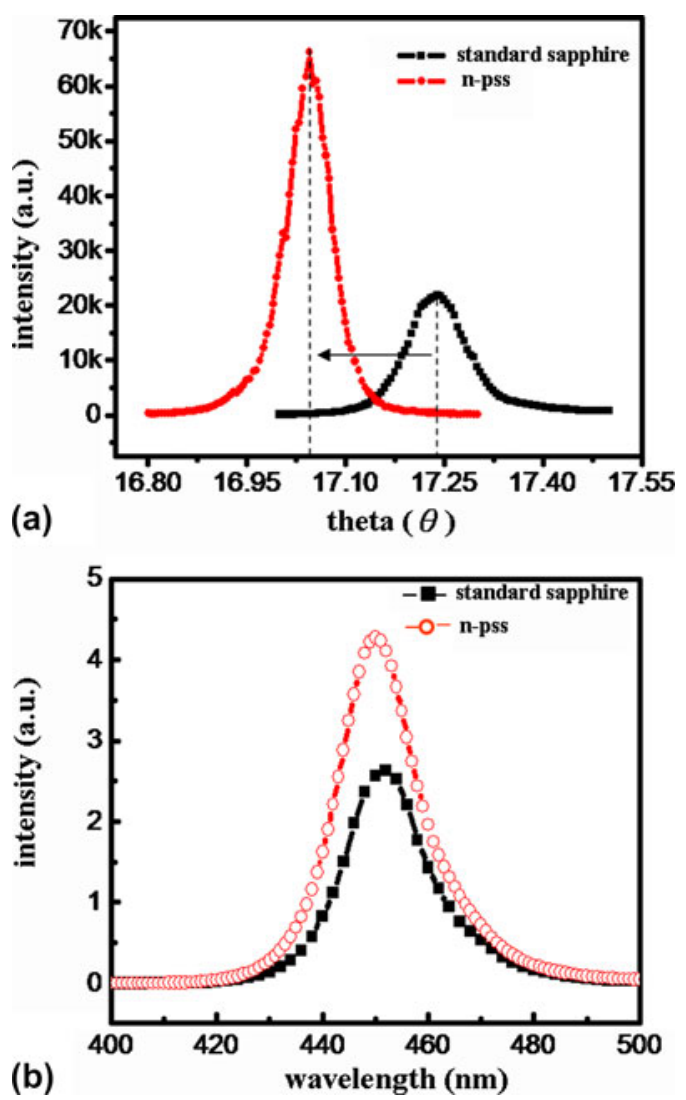

FIG. 5. (a) XRD result and (b) photoluminescence measurement on the GaN epilayer grown on the n-pss and the standard flat sapphire wafers. 
epilayer on the n-pss wafer has a lower defect level (predominant as screw dislocations) than that of the $\mathrm{GaN}$ epilayer on the standard flat sapphire wafer. ${ }^{18}$ Fig. 5(b) shows PL measurement for the GaN epilayers on the n-pss wafer and on the standard flat sapphire wafer, respectively. The GaN epilayer on the n-pss wafer has a higher intensity and a narrower FWHM than that on the standard flat sapphire wafer. Again, the PL results imply that the GaN epilayer grown on the n-pss sapphire wafer has a better epitaxial quality.

The present XRD rocking curve result shows that the (0002) diffraction peak of the GaN epilayer on the n-pss wafer sits slightly toward the smaller angle region, compared to the (0002) diffraction peak of the $\mathrm{GaN}$ epilayer on the standard flat sapphire wafer. Lee et al. ${ }^{19}$ reported a similar finding that the position of the diffraction peak of the GaN epilayer grown on the cone shape-patterned sapphire wafer shifted to the smaller angle region, comparing to that of the $\mathrm{GaN}$ epilayer on the standard flat sapphire wafer. The shift in the (0002) diffraction peak indicates that the in-plane stress level of the $\mathrm{GaN}$ epilayer on the n-pss wafer is different from the in-plane stress level of the GaN epilayer on the standard flat sapphire wafer. So far, there is no study on how the pyramidal patterns on the sapphire wafer would affect the in-plane stress level in the GaN epilayer. In the following, we will calculate the in-plane stress level in the GaN epilayers grown on the $\mathrm{n}$-pss wafer and the standard sapphire wafer. The $d$-spacing values of the (0002) GaN plane on the n-pss wafer and the standard flat sapphire wafer can be determined by Bragg's law:

$$
n \lambda=2 d \sin \theta
$$

where $\lambda$ is the wavelength of $\mathrm{x}$-ray (1.542 $\AA$ ). From XRD rocking curve results, the diffraction angle of the (0002) peak of the GaN epilayers on the n-pss wafer and on the standard flat sapphire wafer is 17.05 and 17.24, respectively. Therefore, plugging in the wavelength of $\mathrm{x}$-ray $(1.542 \AA)$ and the diffraction angles into Bragg's law, the (0002) $d$-spacing of the GaN epilayers on the n-pss wafer and the standard flat sapphire wafer are calculated to be $2.627 \AA\left(d_{\text {n-pss }}\right)$ and $2.598 \AA\left(d_{\text {flat }}\right)$, respectively. Using the interplanar spacing Eq. (2) and the calculated interplanar $d$-spacing values $\left(d_{\text {n-pss }}\right.$ and $\left.d_{\text {flat }}\right)$, the c-axial lattice constants of the GaN epilayers on the n-pss wafer and on the standard flat sapphire wafer can be computed to be $5.254 \AA$ and $5.196 \AA$, respectively.

$$
\frac{1}{d^{2}}=\frac{4}{3}\left(\frac{h^{2}+h k+k^{2}}{a^{2}}\right)+\frac{l^{2}}{c^{2}}
$$

The c-axial lattice constant of the GaN epilayer on the $\mathrm{n}$-pss wafer is larger than that of the GaN epilayer on the standard flat sapphire wafer. Also, we notice that the c-axial lattice constants of both $\mathrm{GaN}$ epilayers are larger than the equilibrium c-axial lattice constant $\left(c_{0}\right)$ of the stress-free bulk GaN ( $c_{0}=5.185 \AA$ ). It means that both $\mathrm{GaN}$ epilayers are in tensile state in the c-axial direction The c-axial strain $\left(\varepsilon_{\mathrm{zz}}\right)$ of the GaN epilayer on sapphire wafer can be expressed as $\varepsilon_{\mathrm{zz}}=\frac{\Delta c}{c_{0}}$, where $\Delta c$ is the difference between the equilibrium c-axial lattice constant $\left(c_{0}\right)$ of the stress-free bulk GaN and the c-axial lattice constant of the GaN epilayer either on the n-pss wafer or on the standard flat sapphire wafer. With knowing the above calculated c-axial lattice constants and the equilibrium c-axial lattice constant of the stress-free bulk GaN $\left(c_{0}=5.185 \AA\right)$, the c-axial strain values $\left(\varepsilon_{\mathrm{zz}}\right)$ of the GaN epilayers on the n-pss wafer and the standard flat sapphire wafer can be calculated to be $1.331 \%$ and $0.212 \%$, respectively. Poisson ratio relates the in-plane compressive strain and the c-axial tensile strain in the GaN epilayer. Then, via the Poisson ratio of $\mathrm{GaN}(v=0.22)$, the in-plane compressive strain values for the $\mathrm{GaN}$ epilayers on the n-pss wafer and the standard flat sapphire wafer are calculated to be $0.268 \%$ and $0.047 \%$, respectively. Using Young's equation, $\sigma=Y \varepsilon,\left(Y_{\mathrm{GaN}}=150 \mathrm{GP}\right)$, the in-plane compressive stress level in the $\mathrm{GaN}$ epilayers on the n-pss wafer and the standard flat sapphire wafer can be calculated to be $305 \mathrm{MPa}$ and $53.6 \mathrm{MPa}$, respectively. The calculated in-plane compressive stress level of the $\mathrm{GaN}$ epilayer on the n-pss wafer is larger than the compressive stress level of the $\mathrm{GaN}$ epilayer on the standard flat sapphire wafer. It means that the pyramidal pattern on the n-pss wafer would enlarge the in-plane compressive stress in the $\mathrm{GaN}$ epilayer. The exact reasons for the augment of the in-plane compressive stress in the GaN epilayer deserve future detail study.

The previous researches have shown that the compressive stress in the GaN epilayer would result in a piezoelectric field across the MQWS, which distorts the energy band diagram and degrades the internal quantum efficiency of the MQWS in GaN LED epilayer. ${ }^{15,16}$ So, it could be expected that, with a larger in-plane compressive stress, the GaN LED epilayer on the n-pss wafer would have a larger degradation effect on the internal quantum efficiency than that of the GaN LED epilayer on the standard flat sapphire wafer.

From the overall discussions above, we realize that the pyramidal pattern on the n-pss wafer would result in two contradiction effects on the internal quantum efficiency of the GaN LED epilayer: (i) the possible lateral growth due to the pyramidal pattern on the n-pss wafer leads to the improvement in the crystalline quality of the MOCVD-grown GaN LED epilayer, which enhances the internal quantum efficiency of the GaN LED epilayer. (ii) Yet, a larger compressive stress level in the GaN epilayer caused by the pyramidal pattern on the n-pss wafer degrades the internal quantum efficiency of the GaN LED epilayer. 


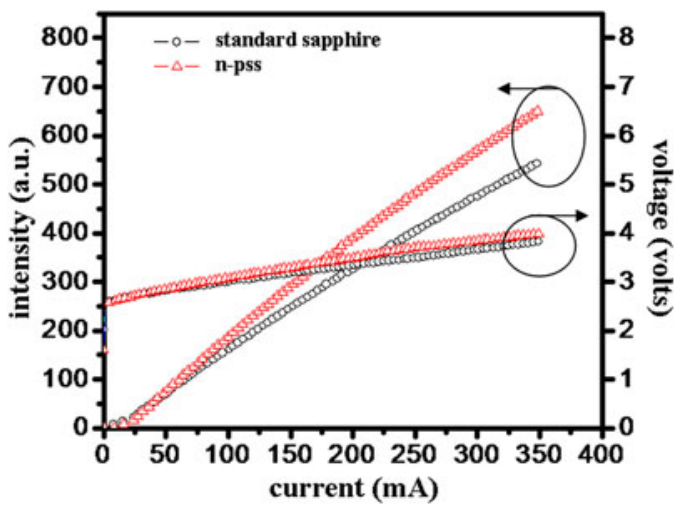

FIG. 6. $L-I-V$ curves of light-emitting diode chips fabricated on the nature-patterned sapphire substrate (n-pss) wafer and on the standard sapphire wafer.

\section{Horizontal LED chip fabrications and characterizations}

Both GaN LED epilayers grown on the n-pss wafer and the standard flat sapphire wafer were fabricated into the conventional horizontal LED chips. Figure 6 is the plot of $L-I-V$ curves of LED chips on the n-pss wafer and the standard flat sapphire wafer. The turn-on voltages of both LED chips on the n-pss wafer and the standard flat sapphire wafer at the $350 \mathrm{~mA}$ input current are very similar (the difference is less than $0.2 \mathrm{~V}$ ). From the $L-I$ curves, we find that the LED chips fabricated on the n-pss wafer have a higher light output than the LED chips on the standard flat sapphire wafer. At the input current of $350 \mathrm{~mA}$, the average light output of the LED chips on the n-pss sapphire wafer is larger than that of the LED chips on the standard flat sapphire wafer by about $20 \%$.

\section{SUMMARY}

In conclusion, three etching solutions [pure $\mathrm{H}_{3} \mathrm{PO}_{4}$, pure $\mathrm{H}_{2} \mathrm{SO}_{4}$, and mixing solution $\left(\mathrm{H}_{3} \mathrm{PO}_{4}: \mathrm{H}_{2} \mathrm{SO}_{4}=3: 1\right)$ ] on sapphire wafers were investigated. Among three kinds of etching solutions, the mixing solution has the fastest etching rate (about $1.5 \mu \mathrm{m} / \mathrm{min}$ ) at $320{ }^{\circ} \mathrm{C}$. The etching rates increases greatly with the etching temperature. On the other hand, it is found that pure $\mathrm{H}_{2} \mathrm{SO}_{4}$ could not etch the sapphire in thickness. Instead, very-facet pyramids were created on the sapphire surface.

XRD and PL measurement show that GaN LED epilayer grown on pyramidal-patterned sapphire wafer has a better crystalline quality than that grown on the standard flat sapphire. In addition, the analysis from the XRD results implies that the pyramidal pattern on the n-pss wafer would enlarge the in-plane compressive stress in the GaN epilayer. Yet, at the input current of $350 \mathrm{~mA}$, the light output of the LED chips on the pyramidal-patterned sapphire is larger than that of the LED chips on the standard sapphire by $20 \%$.

\section{ACKNOWLEDGMENTS}

The authors thank Prof. Chung, National Central University, Taiwan, for the fruitful discussion. Also, the work was financially supported by NSC 100-3113-E-008-001, Top-level Research Centers Grants 100G903-2, and National Science Council Grant projects NSC 98-2221-E008-027-MY3. Also, PL measurement by N.W. Hsu is greatly acknowledged.

\section{REFERENCES}

1. M. Koike, N. Shibata, H. Kato, and Y. Takahashi: Development of high efficiency GaN-based multiquantum-well light-emitting diodes and their applications. IEEE J. Sel. Top. Quantum Electron. 8, 271 (2002).

2. S.H. Lydecker, K.F. Leadford, and C.A. Ooyen: Lighting industry acceptance of solid state lighting. Proc. SPIE Int. Soc. Opt. Eng. 5187, 22 (2004).

3. LEDs Magazine, December 21, 2006. Available at http://ledsmagazine.com/news/3/12/19/1.

4. Y. Narukawa, J. Narita, T. Sakamoto, K. Deguchi, T. Yamada, and T. Mukai: Ultra-high efficiency white light emitting diodes. Jpn. J. Appl. Phys. 45, L1084 (2006).

5. Y.J. Lee, H.C. Kuo, T.C. Lu, B.J. Su, and S.C. Wang: Fabrication and characterization of GaN-Based LEDs grown on chemical wetetched patterned sapphire substrates. J. Electrochem. Soc. 153(12), G1111 (2006).

6. D.S. Wuu, W.K. Wang, W.C. Shih, R.H. Horng, C.E. Lee, W.Y. Lin, and J.S. Fang: Enhanced output power of nearultraviolet InGaN-GaN LEDs grown on patterned sapphire substrates. IEEE Photonics Technol. Lett. 17, 288 (2005).

7. M. Yamada, T. Mitani, Y. Narukawa, S. Shioji, I. Niki, S. Sonobe, K. Deguchi, M. Sano, and T. Mukai: InGaN-based near-ultraviolet and blue-light-emitting diodes with high external quantum efficiency using a patterned sapphire substrate and a mesh electrode. Jpn. J. Appl. Phys. 41, L1431 (2002).

8. Z.H. Feng and K.M. Lau: Enhanced luminescence from GaN-based blue LEDs grown on grooved sapphire substrates. IEEE Photonics Technol. Lett. 17, 1812 (2005).

9. K. Tadatomo, H. Okagawa, Y. Ohuchi, T. Tsunekawa, Y. Imada, M. Kato, and T. Taguchi: High output power InGaN ultraviolet light-emitting diodes fabricated on patterned substrates using metalorganic vapor phase epitaxy. Jpn. J. Appl. Phys. 40(Pt 2), L583 (2001).

10. Y.J. Lee, H.C. Tseng, and H.C. Kuo: Improvement in lightoutput efficiency of AlGaInP LEDs fabricated on stripe patterned epitaxy. IEEE Photonics Technol. Lett. 17(12), 2532 (2005).

11. J.J. Chen, Y.K. Su, C.L. Lin, S.M. Chen, W.L. Li, and C.C. Kao: Enhanced output power of GaN-based LEDs with nano-patterned sapphire substrates. IEEE Photonics Technol. Lett. 20(13), 1193 (2008).

12. P.C. Tsai, W. Ricky, and Y.K. Su: Lifetime tests and junctiontemperature measurement of InGaN light-emitting diodes using patterned sapphire substrates. J. Lightwave Technol. 25(2), 591 (2007).

13. D.S. Wuu, W.K. Wang, K.S. Wen, S.C. Huang, R.H. Horng, Y.S. Yu, and M.H. Pan: Fabrication of pyramidal patterned sapphire substrates for high-efficiency InGaN-based light emitting diodes. J. Electrochem. Soc. 153(8), G765 (2006).

14. F. Dwikusuma, D. Saulys, and T.F. Kuech: Study on sapphire surface preparation for III-nitride heteroepitaxial growth by chemical treatments. J. Electrochem. Soc. 149(11), G603 (2002). 
15. J. Simon, R. Langer, A. Barski, and N.T. Pelekanos: Spontaneous polarization effects in $\mathrm{GaN} / \mathrm{Al}_{\mathrm{x}} \mathrm{Ga}_{1-\mathrm{x}} \mathrm{N}$ quantum wells. Phys. Rev. $B$ 61, 7211 (2000)

16. Y.L. Li, Y.R. Huang, and Y.H. Lai: Efficiency droop behaviors of InGaN/GaN multiple-quantum-well light-emitting diodes with varying quantum well thickness. Appl. Phys. Lett. 91, 181113 (2007).

17. P.T. Törmä, O. Svensk, M. Ali, S. Suihkonen, M. Sopanen, M.A. Odnoblyudov, and V.E. Bougrov: Maskless roughening of sapphire substrates for enhanced light extraction of nitride based blue LEDs. Solid-State Electron. 53, 166 (2009).

18. D.G. Zhao, D.S. Jiang, J.J. Zhu, Z.S. Liu, H. Wang, S.M. Zhang, Y.T. Wang, and H. Yang: Role of edge dislocation and Si impurity in linking the blue luminescence and yellow luminescence in n-type GaN films. Appl. Phys. Lett. 95, 041901 (2009).

19. J.H. Lee, J.T. Oh, Y.C. Kim, and J.H. Lee: Stress reduction and enhanced extraction efficiency of GaN-based LED grown on cone-shapepatterned sapphire. IEEE Photonics Technol. Lett. 20(18), 1563 (2008). 